INVESTIGACIÓN/RESEARCH

\title{
LA COMUNICACIÓN CORPORATIVA A TRAVÉS DE LAS PÁGINAS WEB: EL CASO DE LAS ONGS ESPAÑOLAS
}

Begoña Gómez Nieto1: Universidad Europea Miguel de Cervantes. España mbgomez@uemc.es

Alejandro Tapia Frade: Universidad Europea Miguel de Cervantes. España atapia@uemc.es

Óscar Díaz Chica: Universidad Europea Miguel de Cervantes. España converxa@gmail.com

\section{RESUMEN}

Este trabajo analiza la comunicación corporativa de un centenar de empresas y organizaciones sin ánimo de lucro a través de sus portales Web. Para ello se recurrió a la técnica de análisis de contenido. Los resultados ponen de manifiesto la inadecuada gestión de la comunicación corporativa en este medio, con deficiencias notorias en el desarrollo de las posibilidades de uso del medio. Además, si bien formalmente se da importancia a la comunicación corporativa, también se acusa la ausencia de elementos comunicativos fundamentales y la deficiente ubicación de algunos contenidos que efectivamente consta en las páginas Web.

PALABRAS CLAVE: Comunicación corporativa - Imagen pública - Visibilidad Portal Web - ONG

\footnotetext{
${ }^{1}$ Autor correspondiente:

Begoña Gómez Nieto: Profesora Adjunta en el Grado de Publicidad y Relaciones Públicas de la Universidad Europea Miguel de Cervantes (Valladolid). España.

Correo: $\underline{\text { mbgomez@uemc.es }}$
} 


\title{
CORPORATE COMMUNICATION THROUGH WEB PAGES: THE CASE OF SPANISH NGOS
}

\begin{abstract}
This paper analyzes the corporate communication of a hundred nonprofit organizations through its Web portals. This technique used was the content analysis. The results highlight the inadequate management of corporate communication in this environment, with glaring deficiencies in the development of the usability of the medium. Furthermore, although formally importance is given to corporate communications, also blamed the lack of some basic communicative elements and poor location that actually has some content in Web pages.
\end{abstract}

KEY WORDS: Corporate communication - Public image - Visibility - Web portal NGO.

\section{INTRODUCCIÓN}

La imagen corporativa supone un activo fundamental para las organizaciones, creando valor y estableciéndose como un activo intangible estratégico de primer nivel para la misma, ocupando espacio en la mente de sus públicos, facilitando su diferenciación respecto de organizaciones competidoras, y disminuyendo la influencia de potenciales factores de tipo situacional en el proceso de decisión de compra (Capriotti, 2005:10-11). Dicha imagen se configura a partir de la identidad de la empresa, que tiene dos dimensiones complementarias: la identidad visual o sígnica, que tiene que ver con la forma física de la identidad empresarial, y la dimensión conceptual (Sanz de la Tajada,1996:32), que se relaciona con el comportamiento, la cultura y la personalidad corporativa (Villafañe, 1993:28-29).

Actualmente la imagen corporativa de las empresas en Internet se ha consolidado como un factor estratégico clave en el ámbito empresarial, como demuestra la amplia labor investigadora que se está desarrollando sobre este tema.

Su grado de importancia es de especial relevancia en el caso de las ONGs, más aún después de los últimos escándalos financieros que han rodeado el sector (Diario 20 Minutos, Edición Digital, 09/03/2007 y 03/04/2007), y que en buena medida han mermado su reputación pública, que lógicamente debe ser recuperada. Por ello, entendemos que resulta en todo punto interesante la adecuada gestión de la comunicación corporativa, si lo que se desea es la restauración de la imagen corporativa. Así, estudios de Maiers, Reynolds y Haselkorn (2005) y Haselkorn y Walton (2009) pusieron de manifiesto la importancia de la información y la comunicación en el contexto de las ONGs.

Según los estudios existentes sobre el desarrollo de la comunicación como estrategia 
en las organizaciones no lucrativas, en general, podemos decir que es escaso. En ocasiones, se reduce a acciones de publicidad y marketing para recaudar fondos. Partiendo de este punto, también hay que señalar que muchas organizaciones gestionan su comunicación de manera intuitiva, sin planificar, sin un esquema previo. El problema de no seguir un criterio planificado es que no se pueden ver los resultados, ni se pueden plantear objetivos que supongan un verdadero compromiso para ser eficientes y eficaces en el desarrollo de políticas de comunicación y transparencia. Según Herranz de la Casa (2007), para lograr su supervivencia, las organizaciones tendrán que gestionar adecuadamente su comunicación y ser transparentes en su comportamiento diario". Además dicho autor señala que cualquier escándalo en una ONG supone graves consecuencias para la credibilidad de todo el sector (Herranz de la Casa, 2007).

En un estudio de spots de televisión, Vestergaard (2008) mostró que el discurso de las ONGs en los medios presenta una clara dicotomía: por un lado, es objeto de estrategias cada vez más comerciales con el fin de generar visibilidad, y por otro, manifiesta el escepticismo del público hacia la exposición de moralidad en los medios. Internet supone sin duda un amplio escaparate en el que promover tal imagen. Su ya amplia cobertura, estimado en el 50,5\% de la población española (EGM, 2009:2), hace sugerir la importancia de este medio en la difusión de información en general, siendo por tanto un medio vehicular apto para la gestión de información corporativa.

En ese sentido, Patsioura, Vlachopoulou y Malama (2008) examinaron las actitudes en Malasia frente a las páginas Web corporativas en relación a la comunicación, el feedback obtenido de tal comunicación y finalmente el servicio al cliente como herramientas fundamentales en la política empresarial. Los mismos autores definieron en otro estudio (2007) los factores que tienden a producir en el público efectos comunicativos -esencialmente publicitarios- que posteriormente sirvieron como guía para el desarrollo de webs en criterios como funcionalidad y usabilidad. En otro estudio, Biloslavo y Trnavcevic (2009) señalaron que el posicionamiento corporativo en página Web en base a criterios de ecología y naturaleza no supuso ventaja competitiva en el mercado esloveno.

Por otra parte, Chaudhri y Wang (2007) estudiaron la comunicación de RSC en webs corporativas de empresas tecnológicas de La India. Pudieron constatar la pobre presencia de este elemento en las webs analizadas, y destacaron el no aprovechamiento de los sitios Web para comunicar acerca de este elemento. ConnollyAhern y Broadway (2007) destacaron en otro estudio la falta de aprovechamiento de la capacidad de feedback propia de las webs.

Anteriormente, Esrock y Leichty (2000) destacaron en un estudio sobre 500 webs corporativas que las mismas van dirigidas a múltiples públicos, en concreto inversores, clientes y prensa. Además, jerarquizaron dichos públicos en primarios y secundarios, destacando que la prensa formaba parte de los públicos de orden secundario.

Ya específicamente en el ámbito de las ONGs, en los últimos tiempos se están dando 
experiencias novedosas en Internet. Una de ellas es el proyecto Wikihappiness, una micro-ONG que gestiona su actividad a través de Facebook (Hurst, 2010:34).Vaccaro y Madsen (2008), en un estudio sobre el uso de las nuevas tecnologías y transparencia en una ONG italiana, determinaron que la revelación de información sobre transparencia en estos medios se encontraba bajo la presión de benefactores, inversores y competidores, además de la privacidad que la ONG deseaba tener.

La disparidad de resultados ha impulsado la realización de este trabajo. En el mismo se recoge un estudio sobre la exposición de información corporativa de las ONGs en sus páginas Web, con el objetivo de determinar si dicha comunicación está adecuadamente estructurada en las páginas, y los contenidos son los acertados dentro de dicha estructura.

\section{METODOLOGÍA}

A fin de conocer en qué situación se encuentran las organizaciones sin ánimo de lucro en lo relativo a la comunicación corporativa por Internet, se decidió el uso de la técnica del análisis de contenido con perspectiva cuantitativa. Para ello se realizó una plantilla de análisis compuesta por 60 variables que estructuran e ilustran las posibilidades comunicativas que ofrecen dichas organizaciones en cuanto a comunicación corporativa en Internet.

Se determinó revisar la presencia o ausencia de una sección específica destinada a comunicación corporativa y en su caso en qué nivel estructural se encontraba -medido a través del conteo de clicks necesarios para acceder a dicha sección-. Dentro de este capítulo de información corporativa se examinó si se proporcionaba información de la historia de la institución -y dentro de ésta, si existen referencias explícitas a mitos- , de biografías -y en su caso cuáles- , valores y misión de la organización, organigrama del mismo, calendarios de eventos, informes financieros, información sobre responsabilidad social corporativa, reputación corporativa, desarrollo de marcas, elementos de calidad, elementos de identidad corporativa, etc. También se valoró si en la comunicación se utilizaba únicamente texto o si se aprovechaban las cualidades del medio y se incluía fotografía, audio y video.

Se ejecutó dicha plantilla de análisis sobre los sitios Web de 101 organizaciones sin ánimo de lucro. La recogida de información se efectuó durante el mes de febrero de 2010. A fin de lograr una adecuada representatividad de dichas organizaciones, se seleccionaron dichas organizaciones contando con el presupuesto que habían declarado en sus memorias anuales. Así, se estudiaron organizaciones que ofrecen diferencias notorias a este respecto (en concreto, en un rango que oscila entre los 8.350 euros y los 472 millones de euros, que fueron agrupadas en cuatro grupos: inferior a 100.000 euros, entre 100.001 y 1.000 .000 de euros, entre 1.000.001 y 10.000.000, y finalmente superior a 10.000.000 de euros) teniendo como objetivo ofrecer una visión del sector lo más equilibrada posible. La frecuencia de organizaciones en cada estrato puede apreciarse en la tabla siguiente. 
Tabla 1. Distribución de ONGs analizadas por presupuesto

\begin{tabular}{|l|c|c|c|}
\hline Facturación & Frecuencia & Porcentaje & Porcentaje acumulado \\
\hline Inferior a 100.000 & 21 & 20,8 & 20,8 \\
\hline Entre 100.001 y 1.000.000 & 24 & 23,8 & 44,6 \\
\hline Entre 1.000.001 y 10.000.000 & 38 & 37,6 & 82,2 \\
\hline Superior a 10.000 .000 & 18 & 17,8 & 100 \\
\hline Total & 101 & 100 & \\
\hline
\end{tabular}

Fuente: Elaboración propia a partir de los resultados obtenidos

Se renunció a la proporcionalidad de estratos en este estudio debido a la propia naturaleza del sector: al ser un mercado extremadamente atomizado, con gran número de organizaciones de escaso presupuesto, guardar la debida proporcionalidad implicaría el análisis deficiente de estratos con mayores prepuesto -salvo que se pudiera contar con una muestra muy amplia-. Teniendo en cuenta que uno de los objetivos de este estudio pasa por poner de relevancia diferencias sustanciales en función del presupuesto, y relacionar dichas diferencias con la, a priori, escasa necesidad de presupuesto para gestionar adecuadamente la exposición de estos contenidos, en principio bastante estáticos, se tuvo que renunciar a dicha proporcionalidad.

Para la explotación de datos se utilizó el software específico SPSS v.15.o.

\section{ANÁLISIS Y DISCUSIÓN}

\subsection{Introducción}

La primera consideración acerca de la comunicación en Internet entendemos que debe plantearse en términos dicotómicos, es decir, considerar si efectivamente estas organizaciones tienen o no página Web. Los resultados, tal y como puede apreciarse en la tabla posterior, no dejan lugar a dudas: las ONG tienen, en su mayor parte, página Web, resultando marginal el porcentaje de dichas organizaciones que no la tienen. Por ello, debemos considerar que se tiene, al menos, la posibilidad de exponer información corporativa en este medio sin distinción en razón de presupuesto, lo cual es compatible, a priori, con la accesibilidad en coste de esta herramienta para todas las organizaciones. 
Tabla 2. Porcentaje de organizaciones con página Web estratificado por presupuesto

\begin{tabular}{|l|c|c|c|}
\hline & Tiene página web & & \\
\hline Inferior a 100.000 & Sí & No & Total \\
\hline Entre 100.001 y 1.000.000 & $4,80 \%$ & $95,20 \%$ & $100 \%$ \\
\hline Entre 1.000.001 y 10.000.000 & $4,20 \%$ & $95,80 \%$ & $100 \%$ \\
\hline Superior a 10.000.000 & $2,60 \%$ & $97,40 \%$ & $100 \%$ \\
\hline Total & 0 & $100 \%$ & $100 \%$ \\
\hline
\end{tabular}

Fuente: Elaboración propia a partir de los resultados obtenidos

Otra cuestión es el alcance que se desee tener en este medio. Así, la primera consideración de contenido debe ser en qué idioma o idiomas está disponible dicha página. No obstante, la elección dependerá de forma notoria del público al que se desea alcanzar. En el caso de las ONGs, la elección mayoritaria pasa por el empleo exclusivamente del español, si bien puede apreciarse cierta presencia en el uso del español y la lengua regional correspondiente. Finalmente, el uso de más de dos idiomas (usualmente español, la lengua regional e inglés) es también una opción considerada por estas organizaciones. Los resultados, parecidos en todos los niveles presupuestarios, pueden apreciarse en la tabla 3.

Tabla 3. Porcentaje de uso de idiomas en página Web estratificado por presupuesto

\begin{tabular}{|c|c|c|c|c|c|}
\hline & \multicolumn{4}{|c|}{ ¿En qué idiomas está disponible? } & \multirow[b]{2}{*}{ Total } \\
\hline & $\begin{array}{l}\text { Sólo en } \\
\text { español }\end{array}$ & Español e inglés & Español y otra & $\begin{array}{c}\text { Más de } 2 \\
\text { idiomas }\end{array}$ & \\
\hline & & & $\begin{array}{l}\text { distinta de } \\
\text { inglés }\end{array}$ & & \\
\hline Inferior a 100.000 & $89,50 \%$ & & $10,50 \%$ & & $100 \%$ \\
\hline Entre 100.001 y 1.000 .000 & $55,00 \%$ & $5,00 \%$ & $10 \%$ & $30 \%$ & $100 \%$ \\
\hline $\begin{array}{l}\text { Entre } 1.000 .001 \mathrm{y} \\
10.000 .000\end{array}$ & $75,70 \%$ & & $8,10 \%$ & $16,20 \%$ & $100 \%$ \\
\hline Superior a 10.000 .000 & $72,20 \%$ & $11 \%$ & & $16,70 \%$ & $100 \%$ \\
\hline Total & $73,40 \%$ & $3,20 \%$ & $7,40 \%$ & $16 \%$ & $100 \%$ \\
\hline
\end{tabular}

Fuente: Elaboración propia a partir de los resultados obtenidos

\subsection{La comunicación corporativa en página $W e b$}

Una de las primeras consideraciones a tener en cuenta en la comunicación corporativa en página Web supone entender que si dicha comunicación es importante para la organización ésta deberá ser acreedora de un espacio específico, diferenciado del resto de contenidos, y que además dicho tipo de comunicación ocupará un lugar 
estructuralmente importante en la gestión de contenidos de dicha página. No obstante, es también posible que la comunicación corporativa no sea considerada crucial para las organizaciones, en cuyo caso esta información podría estar tanto subordinada a otros contenidos como, simplemente, no tener presencia en la página Web.

Los resultados indican la preponderante presencia de un espacio específico destinado a la comunicación corporativa, y además ésta se encuentra en un lugar estructural preeminente en la página Web (en término medio, el 91,6\% de las organizaciones sitúa el acceso a un clic de distancia, el 6,3\% presenta dicha información en la página de inicio, y el 2,1\% restante a una distancia de dos clicks, sin haberse encontrado diferencias relevantes en función del presupuesto), lo que, a priori, hace sugerir que estas organizaciones son conscientes de la importancia que tiene para sus públicos la provisión de información corporativa. Los resultados estratificados pueden apreciarse en la tabla posterior.

Tabla 4. Localización de la información corporativa estratificada por presupuesto

\begin{tabular}{|c|c|c|c|c|}
\hline \multirow[t]{2}{*}{ Presupuesto } & \multicolumn{4}{|c|}{$\begin{array}{c}\text { Número de Clicks necesarios para acceder a la sección } \\
\text { corporativa }\end{array}$} \\
\hline & 0 & 1 & 2 & Total \\
\hline Inferior a 100.000 & & $100 \%$ & & $100 \%$ \\
\hline Entre 100.001 y 1.000 .000 & $4,80 \%$ & $90,50 \%$ & $4,80 \%$ & $100 \%$ \\
\hline $\begin{array}{l}\text { Entre } 1.000 .001 \mathrm{y} \\
10.000 .000\end{array}$ & $13,50 \%$ & $83,80 \%$ & $2,70 \%$ & $100 \%$ \\
\hline Superior a 10.000 .000 & $100 \%$ & & $100 \%$ & \\
\hline Total & $6,30 \%$ & $91,60 \%$ & $2,10 \%$ & $100 \%$ \\
\hline
\end{tabular}

Fuente: Elaboración propia a partir de los resultados obtenidos

El título que mayoritariamente se dio a esta sección es en todos los estratos "quiénes somos", presentando el resto de nombres para todos los estratos una presencia muy diversa y marginal -salvo el título "conócenos" para el estrato de más de diez millones de euros, con una presencia del $16,7 \%$ de los casos analizados. Concretamente, el $33,3 \%$ en el estrato inferior, el 45,8\% en el caso de ONGs con presupuesto entre 100.000 y 1.000.000 de euros, el 44,7\% en el estrato de entre 1 y 10 millones de euros, y el 50\% en el estrato de más de 10 millones de euros de presupuesto.

Sin embargo, una cuestión es que se disponga de una sección específicamente destinada a albergar información corporativa, y otra bien distinta es la presencia de contenidos específicos en dicha sección. Por ello, se analizó si existían dichos contenidos, y además si los mismos estaban ubicados en dicha sección, o si por el contrario se encuentran ubicados en otras secciones.

Dentro de éstos, se estudia la presencia y ubicación de biografías en la página Web. Los resultados, expuestos en la tabla siguiente, sugieren la escasa presencia de este elemento en las mismas. En mayor medida se da en las webs con mayor presupuesto, especialmente a partir de un millón de euros. Además, su ubicación es variable, pues 
en algunos casos se sitúan dentro de la sección de información corporativa, y marginalmente en la sala de prensa $\mathrm{u}$ otras ubicaciones. Respecto a quiénes son objeto de relato biográfico, destaca la presencia de presidentes, y vicepresidentes, aunque, como ya se ha mencionado, la presencia de este elemento es muy escaso.

Tabla 5. Items (I) de información corporativa

\begin{tabular}{|c|c|c|c|c|c|c|}
\hline & & \multicolumn{5}{|c|}{ Presupuesto (en euros) } \\
\hline \multicolumn{2}{|c|}{ Items (1) de Información Corporativa } & $\begin{array}{l}\text { Inferior } \\
\mathrm{a} \\
100.000\end{array}$ & $\begin{array}{c}\text { Entre } \\
100.001 \mathrm{y} \\
1.000 .000\end{array}$ & $\begin{array}{c}\text { Entre } \\
1.000 .001 \mathrm{y} \\
10.000 .000\end{array}$ & $\begin{array}{l}\text { Superior a } \\
10.000 .000\end{array}$ & Global \\
\hline \multirow{4}{*}{$\begin{array}{l}\text { ¿Hay biografías } \\
\text { en la web? }\end{array}$} & No & $100,00 \%$ & $90,90 \%$ & $75,00 \%$ & $83,30 \%$ & $85,40 \%$ \\
\hline & Si, en la Sala de Prensa & & & & $11,10 \%$ & $2,10 \%$ \\
\hline & $\begin{array}{l}\mathrm{Si} \text {, fuera de la Sala de } \\
\text { Prensa y dentro de una } \\
\text { sección de Información } \\
\text { corporativa }\end{array}$ & & $4,50 \%$ & $19,40 \%$ & $5,60 \%$ & $9,40 \%$ \\
\hline & $\begin{array}{l}\mathrm{Si}, \text { pero está fuera de la } \\
\text { Sala de Prensa y de la } \\
\text { Información corporativa }\end{array}$ & & $4,50 \%$ & $5,60 \%$ & & $3,10 \%$ \\
\hline \multirow{4}{*}{$\begin{array}{l}\text { ¿Hay Informes } \\
\text { financieros (ej. } \\
\text { Memorias } \\
\text { Anuales)? }\end{array}$} & No & $75,00 \%$ & $36,40 \%$ & $32,40 \%$ & $5,60 \%$ & $37,10 \%$ \\
\hline & Si, en la Sala de Prensa & & & $5,40 \%$ & $11,10 \%$ & $4,10 \%$ \\
\hline & $\begin{array}{l}\text { Si, fuera de la Sala de } \\
\text { Prensa y dentro de una } \\
\text { sección de Información } \\
\text { corporativa }\end{array}$ & & $27,30 \%$ & $35,10 \%$ & $50,00 \%$ & $28,90 \%$ \\
\hline & $\begin{array}{l}\mathrm{Si} \text {, pero está fuera de la } \\
\text { Sala de Prensa y de la } \\
\text { Información corporativa }\end{array}$ & $25,00 \%$ & $36,40 \%$ & $27,00 \%$ & $33,30 \%$ & $29,90 \%$ \\
\hline
\end{tabular}

\section{Fuente: Elaboración propia}

Tendencia distinta puede apreciarse respecto a los informes financieros. Los resultados permiten afirmar que las organizaciones con mayor presupuesto muestran mayor transparencia acerca de su actividad, y además tienden a situar esta información en mayor medida en la sección de información corporativa (la prueba de significación aplicada -con valor 0,000- avala la dependencia de variables, y los indicadores de correlaciones Tau_b de Kendall -con valor 0,479- y Rho de Spearman con valor 0,539- permiten afirmar la relación en sentido directo). Las organizaciones 
de menor presupuesto tienden además a situarlos en lugares ajenos a la información corporativa, siendo por tanto deseable en el caso de estas organizaciones que además de añadir esta información, sea ubicada en lugares próximos a la información corporativa, pues la transparencia también implica facilidad de acceso a la información.

La historia de la institución muestra mayor independencia respecto del presupuesto organizativo, según puede verse en la tabla posterior. Siendo un elemento con escasa presencia en organizaciones con menor presupuesto, cuando es efectiva lo es en la sección de información corporativa. Este hecho también se produce en organizaciones con presupuestos superiores a los 100.000 euros, donde además su presencia es frecuente.

Dentro del relato de la historia, también se analizó la referencia explícita a mitos, al considerarse éstos una formación simbólica de extraordinaria importancia en ese contexto. Si bien suelen tener mayor importancia a nivel interno, son también interesantes cuando en el caso de comunicación externa. Suelen hacer referencia a personas de suma importancia en el desarrollo de la historia organizativa, y usualmente ven incrementadas sus cualidades al extremo de la heroicidad. A nivel interno suponen un referente para los empleados, y son especialmente aptos en organizaciones de este tipo. Así, los datos obtenidos también permiten señalar que la presencia de mitos en el relato de la historia de las organizaciones es más frecuente cuanto mayor es el presupuesto de la ONG (las pruebas de significación bilaterales aplicadas -con valores 0,002 y 0,001- avalan la dependencia de variables, y los indicadores de correlaciones Tau_b de Kendall -con valor 0,423-y Rho de Spearman con valor 0,465- permiten afirmar la relación significativa en sentido directo). En concreto, dicha presencia es muy escasa en organizaciones con presupuestos inferiores a 1.000.000 de euros (de hecho, no se da en el 100\% de los casos inferiores a 100.000 euros y el $85 \%$ de organizaciones con presupuestos inferiores al millón de euros). Sin embargo, su presencia es más frecuente en organizaciones con presupuestos superiores (en concreto, su presencia es un hecho en el $46,2 \%$ de las organizaciones con presupuestos entre 1 y 10 millones de euros, y del 55,5\% de las ONGs con presupuestos superiores a los 10 millones).

Tabla 6. Items (II) de información corporativa

\begin{tabular}{|l|l|c|c|c|c|c|}
\hline \multicolumn{2}{|c|}{} & \multicolumn{5}{c|}{ Presupuesto (en euros) } \\
\cline { 2 - 7 } & $\begin{array}{c}\text { Inferior } \\
\text { a }\end{array}$ & $\begin{array}{c}\text { Entre } \\
100.001 \text { y }\end{array}$ & $\begin{array}{c}\text { Entre } \\
1.000 .001 \\
\text { y }\end{array}$ & $\begin{array}{c}\text { Superior a } \\
10.000 .000\end{array}$ & \\
\multicolumn{2}{|c|}{ Items (2) de Información Corporativa } & 100.000 & 1.000 .000 & 10.000 & Global \\
\hline \multirow{2}{*}{$\begin{array}{l}\text { ¿Está la historia de la } \\
\text { compañía en la web? }\end{array}$} & No & $65,00 \%$ & $13,00 \%$ & $32,40 \%$ & $38,90 \%$ & $35,70 \%$ \\
\cline { 2 - 7 } & Si, en la Sala de & & $4,30 \%$ & $5,40 \%$ & & $3,10 \%$ \\
\hline
\end{tabular}




\begin{tabular}{|c|c|c|c|c|c|c|}
\hline & $\begin{array}{l}\text { Si, dentro de una } \\
\text { sección de } \\
\text { Información } \\
\text { corporativa }\end{array}$ & $35,00 \%$ & $73,90 \%$ & $56,80 \%$ & $61,10 \%$ & $57,10 \%$ \\
\hline & $\begin{array}{l}\text { Si, pero está fuera de } \\
\text { la ambas }\end{array}$ & & $8,70 \%$ & $5,40 \%$ & & $4,10 \%$ \\
\hline \multirow{3}{*}{$\begin{array}{l}\text { ¿Constan los valores y } \\
\text { la misión de la } \\
\text { organización? }\end{array}$} & No & $30,00 \%$ & & $22,20 \%$ & $11,10 \%$ & $16,50 \%$ \\
\hline & $\begin{array}{l}\text { Si, dentro de una } \\
\text { sección de } \\
\text { Información } \\
\text { corporativa }\end{array}$ & $60,00 \%$ & $87,00 \%$ & $75,00 \%$ & $88,90 \%$ & $77,30 \%$ \\
\hline & $\begin{array}{l}\text { Si, pero está fuera de } \\
\text { la ambas }\end{array}$ & $10,00 \%$ & $13,00 \%$ & $2,80 \%$ & & $6,20 \%$ \\
\hline \multirow{3}{*}{$\begin{array}{l}\text { ¿Consta el } \\
\text { organigrama de la } \\
\text { organización en la } \\
\text { web? }\end{array}$} & No & $60,00 \%$ & $65,20 \%$ & $32,40 \%$ & $27,80 \%$ & $44,90 \%$ \\
\hline & $\begin{array}{l}\text { Si, dentro de una } \\
\text { sección de } \\
\text { Información } \\
\text { corporativa }\end{array}$ & $40,00 \%$ & $26,10 \%$ & $62,20 \%$ & $72,20 \%$ & $51,00 \%$ \\
\hline & $\begin{array}{l}\mathrm{Si} \text {, pero está fuera de } \\
\text { ésta y de la sala de } \\
\text { prensa }\end{array}$ & & $8,70 \%$ & $5,40 \%$ & & $4,10 \%$ \\
\hline
\end{tabular}

Fuente: Elaboración propia

La presencia de valores y misión de la institución fueron también analizados. Los datos permiten sugerir una presencia más frecuente que el caso de la historia, y una ubicación preeminente en la sección de información corporativa si bien no se pudo demostrar su relación con el presupuesto ${ }^{2}$. Sin embargo, el organigrama no muestra la misma situación. El análisis realizado sobre dicha variable permite afirmar que su presencia es mayor y su ubicación más acertada cuanto más alto es el presupuesto organizativo (las pruebas de significación bilaterales -con valores 0,004 en ambos casos- avalan la dependencia de variables, y los indicadores de correlaciones Tau_b de Kendall -con valor 0,273- y Rho de Spearman -con valor 0,298- permiten afirmar la relación significativa en sentido directo, aunque menos intensa que en el caso de la presencia de mitos en el relato de la historia), circunstancia que por tanto las de menor presupuesto deberían superar ya que es un elemento en principio estático, y por tanto no requiere atención o mantenimiento frecuente. Sin embargo, hay que señalar que

\footnotetext{
${ }^{2}$ Los indicadores de correlaciones Tau_b de Kendall -con valor 0,070- y Rho de Spearman -con valor 0,076no permiten afirmar la relación entre presupuesto y presencia y ubicación de Misión y Valores.
} 
cuando su presencia es efectiva, su ubicación es a nuestro parecer correcta, pues se encuentra dentro de la sección de información corporativa.

Por otra parte, la existencia de un calendario de eventos en la página Web es un hecho poco frecuente en organizaciones con presupuestos inferiores a 100.000 euros, y algo más frecuente en las demás, como puede apreciarse en la tabla posterior, si bien no pudo establecerse, estadísticamente, relación significativa alguna ${ }^{3}$. Así, su presencia es de aproximadamente la mitad de los casos estudiados de organizaciones con presupuestos superiores a 100.000 euros, aunque su ubicación suele ser ajena a la sección de información corporativa.

Situación distinta es la planteada por el apartado de Responsabilidad Social Corporativa, si bien hay que entender que en el caso de estas organizaciones la diferencia entre información acerca de su actividad y este elemento es muy difusa, pues su tarea principal suele estar estrechamente ligada a las actividades de RSC, llegando en muchos casos a presentar sinergias notables. Como puede verse en la tabla posterior, su presencia es marginal en organizaciones de escaso presupuesto, y muestra cierta presencia en los casos de organizaciones más potentes. Los análisis complementarios realizados permiten afirmar una relación directa estadísticamente significativa entre ambos elementos (los indicadores de correlaciones Tau_b de Kendall -con valor 0,352- y Rho de Spearman -con valor 0,383-, y las pruebas de significación bilateral con valor 0,000 permiten afirmar dicha relación).

Tabla 7. Items (III) de información corporativa

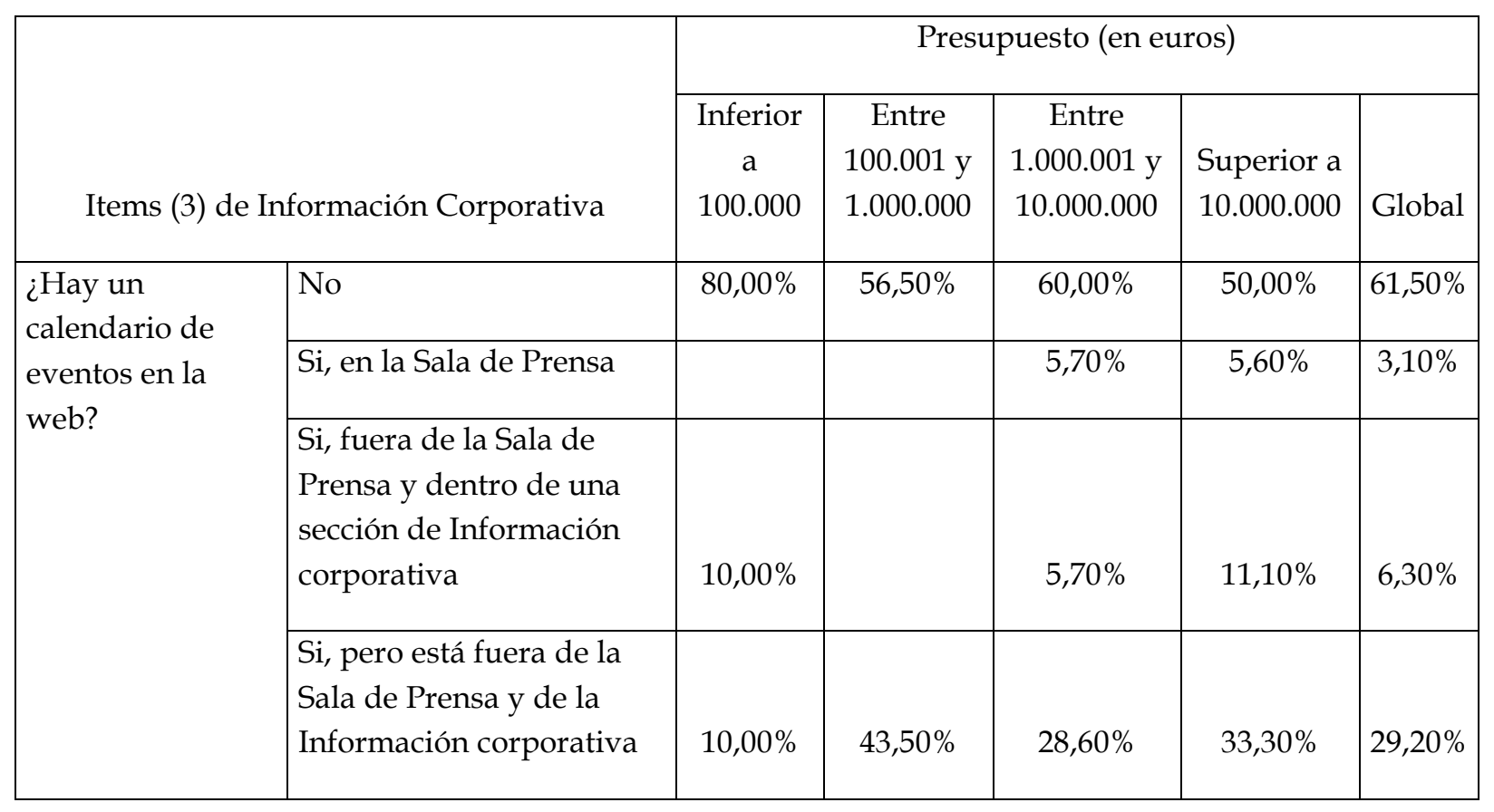

${ }^{3}$ Los indicadores de correlaciones Tau_b de Kendall -con valor 0,131-y Rho de Spearman -con valor 0,150y las pruebas de significación bilateral en 0,145 y 0,150 no permiten afirmar la relación entre presupuesto y presencia y ubicación de calendario de eventos. 


\begin{tabular}{|l|l|c|c|c|c|c|}
\multirow{2}{*}{$\begin{array}{l}\text { ¿Hay un } \\
\text { apartado } \\
\text { específico de } \\
\text { RSC? }\end{array}$} & No & $95,00 \%$ & $91,30 \%$ & $86,50 \%$ & $61,10 \%$ & $84,70 \%$ \\
\cline { 2 - 7 } & $\begin{array}{l}\text { Si, en la sección de } \\
\text { Información corporativa }\end{array}$ & & & $2,70 \%$ & $33,30 \%$ & $7,10 \%$ \\
\cline { 2 - 7 } & $\mathrm{Si}$, en otra sección & $5,00 \%$ & $8,70 \%$ & $10,80 \%$ & $5,60 \%$ & $8,20 \%$ \\
\hline $\begin{array}{l}\text { ¿Hay un } \\
\text { apartado referido } \\
\text { a reputación? }\end{array}$ & No & $100,00 \%$ & $100,00 \%$ & $83,80 \%$ & $72,20 \%$ & $88,80 \%$ \\
\cline { 2 - 7 } & $\begin{array}{l}\text { Si, en la sección de } \\
\text { Información corporativa }\end{array}$ & & & $2,70 \%$ & $27,80 \%$ & $6,10 \%$ \\
\cline { 2 - 7 } & Si, en otra sección & & & $13,50 \%$ & & $5,10 \%$ \\
\hline
\end{tabular}

Fuente: Elaboración propia a partir de los resultados obtenidos

Similar cuestión es la planteada para la reputación corporativa, piedra angular de la transparencia informativa deseable para estas organizaciones. Como puede verse, su presencia es simplemente inexistente en el caso de organizaciones con presupuestos inferiores al millón de euros, e infrecuente en el resto de casos, ubicándose cuando es efectiva su presencia mayoritariamente en la sección de información corporativa.

Del mismo modo el análisis estadístico complementario permite afirmar, al igual que en caso anterior, relación objetiva en sentido directo entre el nivel de presupuesto y la presencia de información sobre reputación (en este caso, los indicadores de correlaciones Tau_b de Kendall -con valor 0,310- y Rho de Spearman -con valor 0,339-, y las pruebas de significación bilateral con valor 0,001 permiten afirmar la misma).

Otros elementos fundamentales para la transparencia informativa son la información sobre calidad en general y procesos de actuación y certificaciones conseguidas en particular. Los datos, expuestos en la tabla inferior, no dejan lugar a dudas: tanto la información específica sobre cuestiones de calidad y procesos de actuación como la información acerca de las certificaciones conseguidas brillan por su ausencia en las páginas Web en todos los casos, salvo en las organizaciones más potentes, donde las cuestiones de calidad y procesos de actuación tienen su lugar en la sección de información corporativa en aproximadamente la mitad de los casos.

Tabla 8. Items (IV) de información corporativa

\begin{tabular}{|l|l|c|c|c|c|c|}
\hline \multirow{2}{*}{} & \multicolumn{5}{c|}{ Presupuesto (en euros) } \\
\cline { 3 - 7 } & Inferior & Entre & Entre & & \\
\multicolumn{2}{|c|}{ Items (4) de Información Corporativa } & a & 100.001 y & 1.000 .001 y & Superior a & \\
& 100.000 & 1.000 .000 & 10.000 .000 & 10.000 .000 & Global \\
\hline ¿Se ofrece información & No & $100,00 \%$ & $95,50 \%$ & $91,40 \%$ & $47,10 \%$ & $86,20 \%$ \\
\hline
\end{tabular}




\begin{tabular}{|c|c|c|c|c|c|c|}
\hline \multirow[t]{2}{*}{$\begin{array}{l}\text { específica sobre } \\
\text { cuestiones de calidad y } \\
\text { procesos de actuación? }\end{array}$} & $\begin{array}{l}\mathrm{Si} \text {, en la sección de } \\
\text { información } \\
\text { coporativa }\end{array}$ & & & & $35,30 \%$ & $6,40 \%$ \\
\hline & $\begin{array}{l}\text { Si ,fuera de la secc. } \\
\text { de información } \\
\text { corporativa y en } \\
\text { otra sección }\end{array}$ & & $4,50 \%$ & $8,60 \%$ & $17,60 \%$ & $7,40 \%$ \\
\hline \multirow[b]{2}{*}{$\begin{array}{l}\text { ¿Se ofrece información } \\
\text { sobre las certificaciones } \\
\text { conseguidas? }\end{array}$} & No & $100,00 \%$ & $100,00 \%$ & $100,00 \%$ & $94,10 \%$ & $98,90 \%$ \\
\hline & $\begin{array}{l}\text { Si, en la sección de } \\
\text { información } \\
\text { coporativa }\end{array}$ & & & & $5,90 \%$ & $1,10 \%$ \\
\hline
\end{tabular}

Fuente: Elaboración propia

Mención aparte requieren otros elementos icónicos al margen de la información puramente textual, como son los símbolos, logotipos o fotografías de edificios o instalaciones, que constituyen una fuente de información irrenunciable en la construcción de discurso e identidad desde una perspectiva corporativa. Por ello, también se incluyó en esta investigación el análisis de la presencia y ubicación tanto de logotipos y símbolos, como fotografías e ilustraciones de edificios e instalaciones.

Los datos resultantes de tal análisis, mostrados en la tabla posterior, permiten sugerir la presencia de logotipos y símbolos en todos los casos analizados con presupuesto superior al millón de euros, aunque en la mayoría de los casos su ubicación es ajena a la sección de información corporativa. En general, este hecho se debe a la presencia sistemática de logotipos y símbolos en la cabecera de las páginas pertenecientes a dichas organizaciones.

Tabla 9. Items (V) de información corporativa

\begin{tabular}{|c|c|c|c|c|c|c|}
\hline & & \multicolumn{5}{|c|}{ Presupuesto (en euros) } \\
\hline \multicolumn{2}{|c|}{ Items (5) de Información Corporativa } & $\begin{array}{l}\text { Inferior } \\
\text { a } \\
100.000\end{array}$ & $\begin{array}{c}\text { Entre } \\
100.001 \mathrm{y} \\
1.000 .000\end{array}$ & $\begin{array}{c}\text { Entre } \\
1.000 .001 \mathrm{y} \\
10.000 .000\end{array}$ & $\begin{array}{l}\text { Superior a } \\
10.000 .000\end{array}$ & Global \\
\hline \multirow{3}{*}{$\begin{array}{l}\text { ¿Hay fotos de } \\
\text { los edificios? }\end{array}$} & No & $89,50 \%$ & $95,20 \%$ & $89,20 \%$ & $44,40 \%$ & $82,10 \%$ \\
\hline & Si, en la Sala de Prensa & $5,30 \%$ & & & & $1,10 \%$ \\
\hline & $\begin{array}{l}\text { Si, fuera de la Sala de } \\
\text { Prensa y dentro de una } \\
\text { sección de Información }\end{array}$ & & $4,80 \%$ & $2,70 \%$ & $22,20 \%$ & $6,30 \%$ \\
\hline
\end{tabular}




\begin{tabular}{|l|l|l|l|l|l|l|}
\hline \multicolumn{1}{|l|}{} & \multicolumn{1}{|l|}{ corporativa } & & & \\
\cline { 2 - 6 } & $\begin{array}{l}\text { Si, pero está fuera de la } \\
\text { Sala de Prensa y de la } \\
\text { Información corporativa }\end{array}$ & $5,30 \%$ & & $8,10 \%$ & $33,30 \%$ & $10,50 \%$ \\
\hline $\begin{array}{l}\text { ¿Tiene la web } \\
\text { logos de la } \\
\text { compañia? }\end{array}$ & No & $15,00 \%$ & $8,70 \%$ & & & $5,10 \%$ \\
\cline { 2 - 7 } & Si, en la Sala de Prensa & & & $2,70 \%$ & $16,70 \%$ & $4,10 \%$ \\
\cline { 2 - 7 } & $\begin{array}{l}\text { Si, fuera de la Sala de } \\
\text { Prensa y dentro de una } \\
\text { sección de Información } \\
\text { corporativa }\end{array}$ & $5,00 \%$ & & & & \\
\cline { 2 - 7 } & $\begin{array}{l}\text { Si, pero está fuera de la } \\
\text { Sala de Prensa y de la } \\
\text { Información corporativa }\end{array}$ & $80,00 \%$ & $91,30 \%$ & $94,60 \%$ & $83,30 \%$ & $88,80 \%$ \\
\hline
\end{tabular}

Fuente: Elaboración propia a partir de los resultados obtenidos

En el caso contrario, el de las organizaciones con presupuestos inferiores al millón de euros, la situación es parecida, si bien no tan intensa. La mayor parte de los casos analizados lo incluyen, pero no todos, por lo que habría que remarcar la importancia de este elemento en la construcción de discurso corporativo. Sin embargo, la presencia de fotografías de edificios e instalaciones parece patrimonio, y sólo parcialmente, de organizaciones con presupuestos superiores a los 10 millones, mostrando además una ubicación variable, aunque tendente a la independencia respecto del resto de información corporativa. En este caso también se pudo constatar relación directa estadísticamente significativa entre presencia de fotografías de edificios y presupuesto organizativo (en este caso, los indicadores de correlaciones Tau_b de Kendall -con valor 0,319- y Rho de Spearman -con valor 0,358-, y las pruebas de significación bilateral con valores 0,001 y 0,000 permiten afirmar la misma).

Sin embargo, otra cuestión es la exposición de contenido mediante audio y vídeo. En lo que respecta al audio, hay que señalar la presencia residual de este elemento en todos los estratos analizados. Además, cuando tiene presencia, la locución se realiza en un único idioma, limitando por tanto el uso de dicho recurso a una comunidad reducida. Por tanto, debemos afirmar que en el caso de los archivos de audio no se aprovecha el potencial de Internet para ofrecer formatos alternativos al texto y la imagen que bien pudieran ser aprovechados por ciertos medios de comunicación, y llegar por tanto a la audiencia que recurre a tales medios para informarse. Los datos pueden apreciarse en la tabla posterior. 
Tabla 10. Items (VI) de información corporativa

\begin{tabular}{|c|c|c|c|c|c|c|}
\hline \multirow{2}{*}{\multicolumn{2}{|c|}{ Vídeo y Audio }} & \multicolumn{5}{|c|}{ Presupuesto (en euros) } \\
\hline & & $\begin{array}{c}\text { Inferior a } \\
100.000\end{array}$ & $\begin{array}{c}\text { Entre } \\
100.001 \mathrm{y} \\
1.000 .000\end{array}$ & $\begin{array}{c}\text { Entre } \\
1.000 .001 \mathrm{y} \\
10.000 .000\end{array}$ & $\begin{array}{l}\text { Superior a } \\
10.000 .000\end{array}$ & Global \\
\hline \multirow{4}{*}{$\begin{array}{l}\text { ¿Hay } \\
\text { vídeos en la } \\
\text { web? }\end{array}$} & No & $60,00 \%$ & $82,60 \%$ & $51,40 \%$ & $27,80 \%$ & $56,10 \%$ \\
\hline & Si, en la Sala de Prensa & & $4,30 \%$ & $10,80 \%$ & $5,60 \%$ & $6,10 \%$ \\
\hline & $\begin{array}{l}\text { Si, dentro de una sección } \\
\text { de información } \\
\text { corporativa }\end{array}$ & & & $2,70 \%$ & $16,70 \%$ & $4,10 \%$ \\
\hline & $\begin{array}{l}\text { Si, pero está fuera de } \\
\text { ambas }\end{array}$ & $40,00 \%$ & $13,00 \%$ & $35,10 \%$ & $50,00 \%$ & $33,70 \%$ \\
\hline \multirow{3}{*}{$\begin{array}{l}\text { ¿Hay } \\
\text { archivos de } \\
\text { audio? }\end{array}$} & No & $90,00 \%$ & $95,70 \%$ & $88,90 \%$ & $94,40 \%$ & $91,80 \%$ \\
\hline & Si, en la Sala de Prensa & & & $5,60 \%$ & & $2,10 \%$ \\
\hline & $\begin{array}{l}\mathrm{Si} \text {, pero está fuera de la } \\
\text { sala de prensa y la } \\
\text { información corporativa }\end{array}$ & $10,00 \%$ & $4,30 \%$ & $5,60 \%$ & $5,60 \%$ & $6,20 \%$ \\
\hline
\end{tabular}

Fuente: Elaboración propia a partir de los resultados obtenidos

La presencia de archivos de vídeo muestra un comportamiento menos uniforme que el antes descrito. Si bien se usa en mayor medida por organizaciones potentes (con presupuesto superior a los 10 millones), en el resto de estratos no muestra un comportamiento uniforme. En cualquier caso, hay que señalar su ubicación usualmente fuera de la sección de información corporativa. En el caso de existir tales vídeos en página Web, éstos van sólo parcialmente provistos de descripciones (concretamente, en el $45,5 \%$ de los casos analizados con presupuestos inferiores a 100.000 euros, un $75 \%$ en el caso de organizaciones de entre 100.000 y 1.000 .000 de euros, un 64,7\% en organizaciones de entre 1 y 10 millones de euros, y un 84,6\% en el caso de ONGs con presupuestos superiores a 10 millones), lo que constituye una evidente falta de atención que debería ser corregida. Las pruebas estadísticas 
complementarias realizadas permiten afirmar relación objetiva estadísticamente significativa entre presupuesto y presencia de vídeo, pero no de audio ${ }^{4}$.

\section{CONCLUSIONES}

Las organizaciones sin ánimo de lucro, independientemente de su presupuesto, muestran una presencia mayoritaria en Internet, lo que implica que al menos poseen el soporte necesario para transmitir información corporativa en dicho medio.

El uso del lenguaje debe considerarse en relación al ámbito geográfico objeto de su actividad. Así, se ha podido constatar la tendencia al uso del español, y en mucha menor medida al uso de ésta y otra lengua, especialmente la regional o el inglés. Habida cuenta de que la mayor parte de organizaciones tienen por lugar de actividad y desarrollo de proyectos España y Sudamérica, parece justificado el uso preponderante del idioma español.

Además, parece que dichas organizaciones han asumido la importancia de la comunicación corporativa en su mix de comunicación, ya que la mayoría tienen una sección específica destinada a albergar estos contenidos, y además ésta se encuentra situada en un lugar estructuralmente preeminente, ya que en la mayoría de los casos es accesible con un único clic.

No obstante, cuestión bien distinta es que dicha sección, específicamente diseñada para albergar contenidos de tipo corporativo, contenga efectivamente este tipo de contenidos, y todavía más, que la totalidad de contenidos corporativos se ubiquen en esta sección.

Así, la presencia de biografías en las páginas Web de estas organizaciones es escasa, y además cuando tiene presencia, muestra una ubicación variable, alejándose por tanto de la deseable situación en la sección de información corporativa. Por otra parte, el relato de la historia de la institución, aún siendo frecuente en organizaciones de más de 100.000 euros de presupuesto, se debería ver incrementado en los restantes estratos. Por otra parte, se ha podido demostrar relación objetiva directa entre la presencia de mitos en el relato de la historia y el presupuesto organizativo. Por ello, entendemos que además de constituir la historia un relato de gran importancia en la elaboración del discurso organizativo, se debería usar con mayor frecuencia la figura del mito, especialmente en organizaciones con presupuestos menores. La presencia de relatos acerca de la misión y valores de la institución es más frecuente que en el caso de la

\footnotetext{
${ }^{4}$ En el caso de vídeo y presupuesto, los indicadores de correlaciones Tau_b de Kendall -con valor 0,211- y Rho de Spearman - con valor 0,232-, y las pruebas de significación bilateral con valores 0,019 y 0,022 permiten afirmar la misma con nivel crítico menor que 0,05 , pero no a nivel 0,01 . En el caso de audio y presupuesto, las pruebas de significación con valores 0,929 y 0,928 eliminan la posibilidad de establecer relación objetiva. Además, las correlaciones con valores $-0,008$ y -0,009 nos acercan a la consideración de independencia de estas variables.
} 
historia, y además suele estar mejor situado, lo cual supone un acierto en tanto que su presencia constituye la declaración de el qué y el cómo de la organización.

Otros elementos que aportan información corporativa son la presencia de organigramas organizativos y calendarios de actividades. El primero de ellos muestra una relación objetiva directa con el presupuesto organizativo. Siendo un elemento usualmente bastante estático, parece por tanto susceptible de ser incluido con independencia de los recursos que se tengan. El calendario de actividades es, al contrario, un elemento que naturalmente debería sostener fuerte dinamismo, por lo que parece comprensible que organizaciones de escasos recursos no tengan la posibilidad de incluirlo, al carecer usualmente de recursos para gestionar tal información, pero su ausencia no parece justificable en el caso de organizaciones con presupuestos elevados. Además, la ubicación de éste último elemento no suele encontrase en la sección de información corporativa.

La memoria anual creemos que constituye un elemento de singular importancia en la comunicación corporativa de las organizaciones, pues en buena medida justifica la propia existencia de las mismas, al ser un documento que explica lo realizado durante un año. Resulta por tanto fundamental si la transparencia informativa es una meta. A este respecto, se ha podido demostrar que cuanto mayor es el presupuesto organizativo, existe mayor probabilidad de que la memoria anual esté presente. No obstante, sería deseable que más organizaciones lo incorporaran, con la excepción de aquellas con mayores recursos, que casi en su totalidad disponen de la misma. Otros elementos interesantes para el logro de transparencia informativa son las informaciones referentes a cuestiones de calidad y procesos de actuación, que sin embargo, en general, no son atendidas con la debida diligencia.

Los elementos icónicos, tales como símbolos, logotipos, imágenes de edificios o instalaciones constituyen también una valiosa fuente de generación de discurso organizativo. A ese respecto, hay que señalar que estas organizaciones usan en gran medida en sus páginas Web logotipos y símbolos, pero no se da igual situación en el caso de fotografías de edificios e instalaciones, ya que únicamente las organizaciones con mayores recursos suelen incorporarlas (en este caso también se demostró relación directa estadísticamente significativa entre presupuesto organizativo y probabilidad de presencia de tal tipo de fotografía).

Sin embargo, el uso de vídeos parece patrimonio exclusivo de organizaciones con presupuestos abultados, y el audio es simplemente testimonial en todos los estratos, lo que supone el desperdicio sistemático del potencial comunicativo que este medio otorga.

En definitiva, debemos concluir la inadecuada gestión de la comunicación corporativa en este medio, ya que en primer lugar consideramos que se desaprovecha la flexibilidad de soportes que le es inherente, al contener información mayoritariamente textual y prescindir de otras opciones. Además, aunque la presencia de una sección específica en las páginas analizadas es mayoritaria, la gestión de contenidos de dicha 
sección no puede calificarse de buena, ya que la misma no contiene toda la información que debiera, y todavía más, existen contenidos en otras partes de la misma página que sería deseable tuvieran su presencia en dicha sección.

Por todo ello, consideramos que estas organizaciones no aprovechan el potencial que este canal tiene -que combina un generalmente bajo coste de implantación de la información con un elevado margen de explotación estratégica-, y con ello se desperdicia la posibilidad de desarrollar en mayor medida la comunicación corporativa de estas organizaciones.

\section{REFERENCIAS}

Biloslavo, R. y Trnavcevic, A. (2009). "Web sites as tools of communication of a green company". En: Management Decision, vol. 47, nº 7 p.1158-1173.

Capriotti, P. (2005). Planificación estratégica de la imagen corporativa. Barcelona: Ariel Comunicación.

Chaudhri, V. y Wang, J. (2007). "Communicating corporate social responsibility on the internet - A case study of the top 100 information technology companies in India". En: Management Communication Quarterly, vol. 21, $\mathrm{n}^{\circ}$ 2, p. 232-247.

COnnolly-Ahern, C. y Broadway, C. (2007). "The importance of appearing competent: An analysis of corporate impression management strategies on the World Wide Web". En: Public Relations Review , vol. 33, nº 3, p.343-345.

Diario 20 Minutos (2007). Detenido el presidente de la ONG Anesvad por apropiación indebida de fondos. Edición Digital, 9 de Marzo. Disponible en http://www.20minutos.es

Diario 20 Minutos (2007). Anticorrupción investiga a Intervida por el posible de desvío de fondos de apadrinamientos para fines privados. Edición Digital, 3 de Abril. Disponible en http://www.20minutos.es

Esrock, S.L. y Leichty, G.B. (2000). “Organization of corporate web pages: Publics and functions". En: Public Relations Review, vol. 26, n 3, p. 327-344.

Estudio General de Medios (E.G.M.) (2009). Estudio de la audiencia de internet. Octubre / Noviembre 2009. Disponible en http://www.aimc.es

Fundación Eroski (2005). Las ONGs ordenadas por presupuesto. Disponible en http://ong.consumer.es/ por-presupuesto/?f $=10000000$

Haselkorn, M. y Walton, R. (2009). “The Role of Information and Communication in the Context of Humanitarian Service". En: IEEE transactions on professional Communication, vol. 53, $\mathrm{n}^{\mathrm{o}} 4$, p.325-328. 
Herranz De La Casa, J.M. (2007). Disponible en:

http:/ / www.consumer.es/web/es/solidaridad/proyectos_y_campanas/2007/08/22 /166049.php (7/06/11).

Hurst, M. (2010) “Wikihappiness, un modelo de ONG basada en las redes sociales". Yorokubu, vol. 3, p. 34.

Maiers, C., Reynolds, M. y Haselkorn, M. (2005). "Challenges to effective information and communication systems in humanitarian relief organizations". En IEEE International Professional Communication Conference (IPCC), p.82-91.

Patsioura, F., Vlachopoulou, M. y Malama, E. (2007). “Corporate advertising web sites - Effectiveness in terms of promotion, communication and relationship marketing". En: ICE-B 2007: Proceedings of the second international conference on e-Business, p. 301306.

Patsioura, F., Vlachopoulou, M. y Malama, E. (2008). “Corporate Advertising Web Sites as Integrated Relationship Marketing Mediums: Effects of Communication, Feedback and Customer Service on Advertising Effects". En: Innovation and knowledge management in business globalization: theory \& practice, Vols 1 y 2 , p. 1336-1341.

Sanz De La Tajada, L.A. (1996). Auditoría de la imagen de la empresa. Métodos y técnicas de estudio de la imagen. Madrid: Síntesis.

Soria, M.M. (2009). “Cómo las ONGs españolas afrontan una crisis de imagen a través de la sala de prensa virtual". En: Razón y Palabra, vol. 70.

Vaccaro, A. y Madsen, P. (2008). "ICT and an NGO: Difficulties in attempting to be extremely transparent". En: Transparency, Information and communication technology: social responsibility and accountability in business and education. Transparency and Social Responsibility Conference Catholic Univ Lisbon, Sch Business \& Econ, Lisbon. p. 5168.

Vestergaard, A. (2008). "Humanitarian branding and the media The case of Amnesty International". En: Journal of language and politics, vol. 7, n 3. p. 471-493.

Villafañe, J. (1993). Imagen positiva. Gestión estratégica de la imagen de las empresas. Madrid: Pirámide.

\section{Begoña Gómez Nieto}

Doctora en Ciencias de la Información por el IE Universidad de Segovia. Licenciada en Publicidad y Relaciones Públicas por el Colegio Universitario de Segovia. Profesora adjunta en la Licenciatura y en el Grado de Publicidad y Relaciones Públicas de la Universidad Europea Miguel de Cervantes de Valladolid. Dirección de numerosos 
proyectos de fin de carrera de alumnos de Periodismo y de Publicidad y Relaciones Públicas. Co-directora del curso "Nuevas tendencias de comunicación en la empresa" (2011) en la UEMC y la dirección del taller para el "I Curso de Verano Periodismo y Comunicación para la salud" (2008) en la UEMC. Directora de la Comisión de Publicidad y Relaciones Públicas en la adaptación de los planes de estudios al Espacio Europeo de Educación Superior (EEES). Entre sus publicaciones destacan varios artículos centrados en el ámbito de la comunicación corporativa, comunicación publicitaria y nuevas formas de comunicación publicitaria (ambient marketing, flagships, redes sociales, estructura de contenidos en webs,...). Autora del libro "Las herramientas de comunicación en la internacionalización de la pyme segoviana".

\section{Alejandro Tapia Frade}

Licenciado (1999) en Publicidad y Relaciones Públicas y Doctor (2005) por el Departamento de Comercialización e Investigación de Mercados, ambos en la Universidad Complutense de Madrid. Profesor adjunto en la Universidad Europea Miguel de Cervantes en la licenciatura de Publicidad y Relaciones Públicas, concretamente en las asignaturas de Teoría de Relaciones Públicas, Sistemas y procesos de la Publicidad y las Relaciones Públicas, Patrocinio y Mecenazgo e Investigación de Mercados. Director de Proyectos de Fin de Carrera. Acreditación por parte de la Agencia para la Calidad del Sistema Universitario de Castilla y León (ACSUCYL) a la figura de Profesor de Universidad Privada. Adicionalmente, ha realizado intercambios docentes en las Universidades de Leiria y Guarda, ambas en Portugal, con una beca ERASMUS. Sus líneas de investigación se hallan en relación con la publicidad y la tecnología, especialmente internet (páginas web y redes sociales) y videojuegos.

\section{Óscar Díaz Chica}

Licenciado en Publicidad y Relaciones Públicas, y en Humanidades, y Diplomado en Estudios Avanzados en Comunicación, Auge Tecnológico y Renovación Sociocultural. Profesor en la Facultad de Comunicación de la Universidad San Jorge. Ha impartido docencia universitaria sobre Publicidad, Relaciones públicas, Creatividad, Planificación de Medios, Marketing y Publicidad Exterior. Coordinador de Proyectos de Fin de Carrera. Su inquietud investigadora ha estado relacionada con la formación de la identidad personal a través de los medios de comunicación y la integración de la responsabilidad social de un modo rentable en el modelo de negocio empresarial. 